\title{
A previsibilidade biométrica nas lentes intra-oculares multifocais
}

\section{Biometric predictability in multifocal intraocular lens}

Augusto Cézar Lacava', Juan Carlos Caballero', Virgilio Centurion'

\section{Resumo}

Objetivo: Apresentar a previsibilidade biométrica, avaliando a refração pós-operatória e acuidade visual (AV), em olhos submetidos a implante de lente intra-ocular multifocal. Métodos: Estudo retrospectivo em 88 olhos de 50 pacientes, submetidos à facoemulsificação com implante de lentes multifocais, de três marcas comerciais ReSTOR, ReZoom e Tecnis. No exame biométrico foram utilizados a biometria óptica ou biometria por interferometria de baixa coerência, associada ao uso de tomógrafo de segmento anterior e à fórmula biométrica Holladay II. Com a refração pós-operatória, acuidade visual e a dioptria da LIO implantada, segundo a fórmula Holladay II, calculamos o valor da lente intra-ocular (LIO) a ser implantada caso utilizássemos as fórmulas SRKT e Haigis. Os resultados foram avaliados por métodos estatísticos. Resultados: Todos os olhos obtiveram AV igual ou maior a 20/40 e J sem correção. As fórmulas Holladay II, SRKT e Haigis apresentaram performance semelhante em todos os grupos de olhos. Conclusão: A utilização de novas tecnologias, como a biometria óptica e fórmulas de última geração, favorecem a obtenção do resultado refracional almejado com grande previsibilidade.

Descritores: Lentes intra-oculares; Acuidade visual; Biometria/métodos

\footnotetext{
'Oftalmologistas do Instituto de Moléstias Oculares - IMO - São Paulo (SP), Brasil.

Trabalho realizado no Instituto de Moléstias Oculares - IMO - São Paulo (SP), Brasil.

Recebido para publicação em: 23/05/07 - Aceito para publicação em 27/08/07
} 


\section{INTRODUÇÃO}

A $\mathrm{s}$ lentes multifocais ou de focos múltiplos podem ser agrupadas em duas categorias: asrefrativas e as difrativas ${ }^{(1)}$.

A ReZoom - AMO é uma LIO refrativa com 5 anéis programados para propiciar boa acuidade visual ao longe, para região intermediária e para perto; estes anéis são uma modificação da multifocal Amo Array ${ }^{(2)}$.

A ReSTOR - Alcon é uma LIO com a parte óptica refrativa - difrativa apodizada. A apodização é a redução gradativa da altura dos degraus dos anéis, proporcionando um melhor gerenciamento da luz dentro do olho com melhor acuidade visual para longe e perto, e razoável visão intermediária ${ }^{(2)}$.

A Tecnis - AMO é uma LIO criada a partir de estudos das aberrações das frentes de ondas, sendo considerada uma LIO asférica negativa que utiliza o princípio difrativo na superfície posterior da $\mathrm{LIO}^{(2)}$.

Estas LIOs exigem, além de uma técnica cirúrgica apurada, uma biometria com muita acurácia, visando à obtenção da emetropia, entretanto há uma margem de erro de 20,3\% no cálculo de uma LIO, quando é colocada uma constante diferente da que foi calculada previamente e um erro de 7,89\% na seleção de uma fórmula inadequada ${ }^{(3)}$.

O objetivo do presente estudo é apresentar a previsibilidade biométrica, avaliando a refração e AV pós-operatória com a fórmula Holladay II, e compará-la com as fórmulas SRKT e Haigis, em olhos com implantes de LIOs de focos múltiplos.

\section{Métodos}

Estudo retrospectivo de 50 pacientes, com idade entre 44 e 87 anos, sendo 26 do sexo feminino (66,6\%), em que foram avaliados 88 olhos portadores de catarata, submetidos à facoemulsificação com implante de lentes intraoculares de focos múltiplos. Os 12 olhos restantes não tinham indicação cirúrgica e não eram portadores de catarata. Os olhos foram divididos em três grupos conforme a LIO utilizada:1) ReSTOR;2) ReZoom e 3) Tecnis. Todas as cirurgias foram realizadas pelo mesmo cirurgião (VC).

A medida do comprimento axial do globo ocular, da ceratometria, da profundidade da câmara anterior e do diâmetro horizontal corneano foi obtida com o uso do biômetro óptico ou biometria por interferometria de baixa coerência (IOL Master Zeiss). A medida da espessura do cristalino foi obtida pelo tomógrafo do segmento anterior (Pentacam - Oculus). A fórmula utilizada para o cálculo do poder dióptrico da LIO foi a de Holladay II. Obtida a refração pós-operatória, AV e biometria pré-operatória, calculamos o valor da LIO a ser implantada utilizando a fórmula SRKT ou a fórmula de Haigis e a comparamos com a Holladay II.

Os dados obtidos foram submetidos a tratamento estatístico, com o objetivo de verificar a eficiência das três formulas. Utilizamos os métodos estatísticos de Bland e Altman e o coeficiente de concordância (modelo de Lin), comparando em cada LIO, as três fórmulas. A medida das diferenças entre os dois métodos (viés) e intervalos de confiança $95 \%$, ou seja, um intervalo onde não há variação em medidas repetidas do mesmo ponto. A definição matemática do que é limite de concordância e a construção matemática do intervalo de confiança para esta estimativa pontual encontra-se em artigo de referência de nossa bibliografia ${ }^{(4)}$. Os intervalos de confiança foram calculados para os coeficientes de concordância de Lin e para os coeficientes de correlação linear de Pearson. Todas as probabilidades de significância (valores de $\mathrm{p}$ ) apresentadas são do tipo bilateral e os valores menores que 0,05 considerados, estatisticamente significantes. As proporções foram comparadas em tabelas de contingência utilizando-se o teste FisherFreeman-Halton e os valores de p menores que 0,05 foram considerados, estatisticamente significantes.

\section{Resultados}

A tabela 1 mostra as características genéricas por grupos.

O resultado refracional do grupo I (ReSTOR) se encontra na tabela 2. Todos os olhos obtiveram acuidade visual sem correção de 20/40 ou melhor para longe e $\mathrm{J}_{3}$ para perto, sendo que 14 olhos obtiveram AV 20/20 e $\mathrm{J}_{1}$ sem correção (tabela 3 ). Nos olhos com refração plana (23 olhos), a fórmula SRKT obteve acerto de 50,00\%, Holladay II em 50,00\% e Haigis em 38,88\%.

O resultado refracional do grupo II (ReZoom) se mostra na tabela 4. Acuidade visual de $20 / 20$ e $\mathrm{J}_{2}$ sem correção em 3 olhos (tabela 5). Todos os pacientes obtiveram AV 20/40 ou melhor para longe e $J_{3}$ para perto sem correção (tabela 6). Nos olhos com refração plana, a fórmula Holladay II obteve 50,00\% de acertos, 33,3\% com SRKT e $16,6 \%$ com Haigis.

O resultado refracional do grupo III (Tecnis) se encontra na tabela 7. Todos os pacientes obtiveram AV de 20/40 ou melhor para longe e $\mathrm{J}_{2}$ para perto sem correção (tabela 8). A fórmula Holladay II obteve 100,00\% dos acertos nos olhos com refração plana.

Tentamos estabelecer se uma fórmula é mais adequada que outra para os diversos tipos de lentes intraoculares ${ }^{(5-6)}$.

Com relação à previsibilidade biométrica em que o resultado foi refração plana (tabela 8), observa-se que a fórmula Holladay II foi responsável pelo acerto de $50,00 \%$ no grupo I e II e 100,00\% no grupo III, ou seja, conseguimos refração plana em praticamente $50,00 \%$ de todos os olhos submetidos a implante de LIO multifocal. Com relação à fórmula SRKT, obtivemos refração plana em 50,00\% dos casos do grupo I e 33,30\% 
Tabela 1

Características genéricas dos grupos

\begin{tabular}{lrrr}
\hline Características & ReSTOR & ReZoom & Tecnis \\
\hline Pacientes & 28 & 10 & 12 \\
Olhos & 50 & 18 & 20 \\
Idade & & & 68,50 \\
Média & 70,23 & 61,30 & $48-81$ \\
Variação & $56-87$ & $32-80$ & 5 \\
Sexo Masculino & 10 & 5 & 7 \\
Sexo Feminino & 18 & 5 & $+0,32$ \\
Equivalente esférico pós-operatório & & & \\
Média & $+0,19$ & $+0,22$ & Plana $+1,5$ \\
Variação & Plana $+1,0$ & & 0,33 \\
Cilindro pós-operatório & 0,28 & Plana $-1,00$ & Plana $-1,0$ \\
Média & Plana $-1,75$ &
\end{tabular}

Tabela 2

\section{Refração pós-operatória grupo ReSTOR}

\begin{tabular}{ccr}
\hline Refração & $\mathbf{N}^{\mathbf{0}}$ de Olhos & \multicolumn{1}{c}{$\%$} \\
\hline Plana & 23 & 46,00 \\
EE Plano & 3 & 6,00 \\
EE +0.25 & 9 & 18,00 \\
EE +0.50 & 12 & 24,00 \\
EE +0.75 & 3 & 6,00 \\
Total & 50 & 100,00 \\
\hline
\end{tabular}

Tabela 4

Refração pós-operatória grupo ReZoom

\begin{tabular}{ccr}
\hline Refração & $\mathbf{N}^{\mathbf{0}}$ de Olhos & \% \\
\hline Plana & 6 & 33,33 \\
EE Plano & 2 & 11,11 \\
EE +0.25 & 3 & 16,67 \\
EE +0.50 & 7 & 38,89 \\
EE +0.75 & 0 & 0,00 \\
Total & 18 & 100,00 \\
\hline
\end{tabular}

Tabela 6

Previsibilidade de refração plana nos três grupos com as três fórmulas

\begin{tabular}{|c|c|c|c|}
\hline Fórmula $\quad$ LIO & ReSTOR & ReZoom & Tecnis \\
\hline Holladay II & $50,00 \%$ & $50,00 \%$ & $100,00 \%$ \\
\hline SRKT & $50,00 \%$ & $33,00 \%$ & \\
\hline Haigis & $38,88 \%$ & $16,6 \%$ & \\
\hline
\end{tabular}

Tabela 3

Av com e sem correção pós-operatória grupo ReSTOR

\begin{tabular}{lcrrr}
\hline AV & SC & \multicolumn{1}{c}{$\%$} & CC & \multicolumn{1}{c}{$\%$} \\
\hline 20/20 J1 & 14 & 28,00 & 30 & 60,00 \\
20/30 J1 J2 & 19 & 38,00 & 18 & 36,00 \\
20/40 J3 & 17 & 34,00 & 2 & 4,00 \\
Total & 50 & 100,00 & 50 & 100,00 \\
\hline
\end{tabular}

Tabela 5

Av com e sem correção pós-operatória grupo ReZoom

\begin{tabular}{lcrrr}
\hline AV & SC & \multicolumn{1}{c}{$\%$} & CC & \multicolumn{1}{c}{$\%$} \\
\hline 20/20 J1 & 3 & 16,67 & 13 & 72,22 \\
20/25 J1 & - & - & 2 & 11,11 \\
20/30 J2 & 5 & 27,78 & 3 & 16,67 \\
20/40 J3 & 10 & 55,55 & - & - \\
Total & 18 & 100,00 & 18 & 100,00 \\
\hline
\end{tabular}

Tabela 7

Refração pós-operatório grupo Tecnis

\begin{tabular}{ccr}
\hline Refração & $\mathbf{N}^{\mathbf{0}}$ de Olhos & \% \\
\hline Plana & 6 & 30,00 \\
EE Plano & 1 & 5,00 \\
$\mathrm{EE}+0.25$ & 4 & 20,00 \\
$\mathrm{EE}+0.50$ & 7 & 35,00 \\
$\mathrm{EE}+1.00$ & 2 & 10,00 \\
Total & 20 & 100,00 \\
\hline
\end{tabular}


Tabela 8

\section{Av com e sem correção pós-operatória grupo Tecnis}

\begin{tabular}{lcrrr}
\hline AV & SC & \multicolumn{1}{c}{$\%$} & CC & \multicolumn{1}{c}{$\%$} \\
\hline $20 / 20 \mathrm{~J} 1$ & 6 & 30,00 & 19 & 95,00 \\
$20 / 25 \mathrm{~J} 1$ & - & - & - & - \\
$20 / 30 \mathrm{~J} 2$ & 8 & 40,00 & 1 & 5,00 \\
$20 / 40 \mathrm{~J} 3$ & 6 & 30,00 & - & - \\
Total & 20 & 100,00 & 20 & 100,00 \\
\hline
\end{tabular}

do grupo II. Com relação à fórmula Haigis, a refração plana foi conseguida em $38,88 \%$ no grupo I e $16,60 \%$ no grupo II. A aparente discrepância entre as fórmulas se deve ao fato de compararmos só os pacientes com refração plana, já análise estatística avalia todos os olhos e todos os grupos e em todas as fórmulas mostrou distribuição de padrão semelhante (gráficos 1, 2 e 3).

Ao avaliar a previsibilidade refracional e à refração plana acrescentarmos o equivalente esférico de até 0.50 dioptrias (tabelas 2, 4 e 7), podemos concluir com os seguintes resultados de previsibilidade: no grupo I: $94,00 \%$, no grupo II: $100,00 \%$ e no grupo III: $90,00 \%$ de previsibilidade.

A análise estatística mostrou que as três fórmulas apresentam performances semelhantes. $\mathrm{O}$ tratamento estatístico mostrou que o coeficiente de concordância de todas as fórmulas foi maior que 0,90 , definido como excelente concordância. Este coeficiente é o mais adequado para amostras pequenas (gráficos 1,2 e 3). Ou seja, as três fórmulas apresentam performance semelhante.

\section{Discussão}

As lentes intra-oculares de focos múltiplos apresentam-se hoje como uma opção para a população cuja longevidade tem crescido e por isso está mais produtiva e mais exigente quanto a sua qualidade de vida.

Em um estudo realizado, em 1998, com a lente multifocal Amo Array, observamos 113 pacientes, e em 37 os implantes foram bilaterais e se obteve $86,14 \%$ dos olhos com AV 20/40 ou melhor para longe e em 92,48\% de $\mathrm{J}_{3}$ para perto sem uso de correção óptica ${ }^{(7)}$. Naquele momento, utilizávamos biometria ultra-sônica por aplanação e fór-

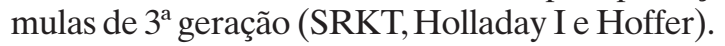

No presente estudo, utilizamos fórmulas biométricas de $4^{\mathrm{a}}$ geração (Holladay II e Haigis) e de $3^{\mathrm{a}}$ geração (SRKT) e realizamos biometria óptica na quase totalidade dos procedimentos. Apenas no olho número 20 do grupo ReSTOR e nos de n 12 , 13 e 16 do grupo ReZoom foram realizados biometria ultra-sônica por aplanação, devido à opacificação cristaliniana não permitir o uso da biometria óptica. A utilização do IOL Master otimiza o tempo do exame, mas não permite ficar independente do ultra-som. No momento desta publicação, a Zeiss está apresentando um novo software do
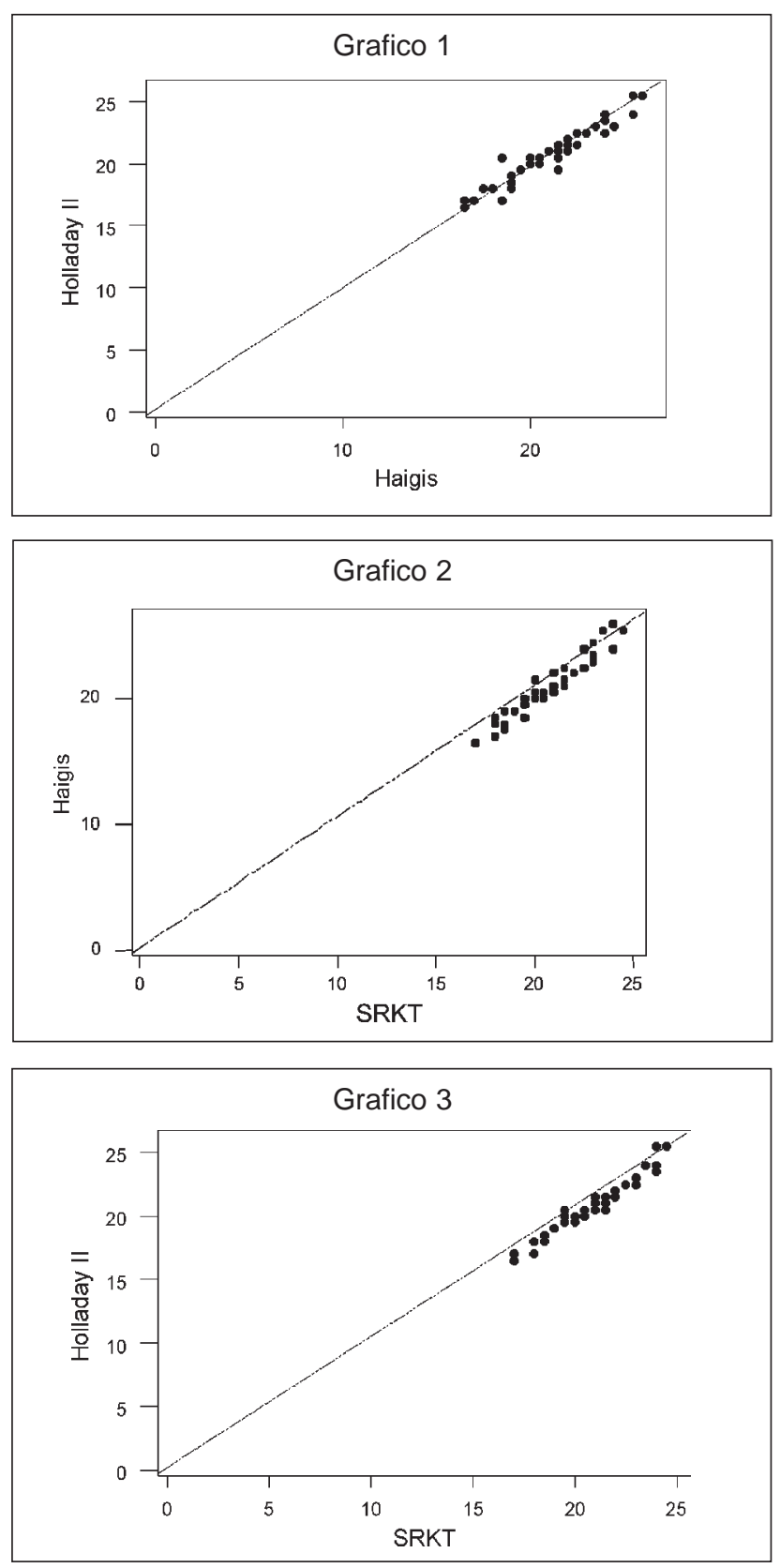

IOL Master que visa à eficiência de medidas nas opacidades cristalinianas mais avançadas.

Um trabalho ${ }^{(8)}$, comparando a biometria ultrasônica por aplanação e interferometria, mostra que o biometrista experiente pode apresentar resultados semelhantes à interferometria. A literatura afirma que a biometria ultra-sônica por imersão mostra medidas tão confiáveis quanto à biometria óptica ${ }^{(9)}$.

Quanto à refração pós-operatória, observamos que no estudo com Amo Array 54,12\% apresentaram refração plana, 22,62\% refração entre $\pm 0,50$ dioptria e $23,26 \%$ refração maior que 0,75 dioptria $^{(7)}$. 
Ao compararmos os 2 estudos com multifocais, observamos que a previsibilidade e acurácia da biometria vêm melhorando paulatinamente, ou seja, obtivemos em $100 \%$ dos olhos AV 20/40 e J - J ou melhor e a refração entre plana e \pm 0.50 em $94,00 \%$ dos implantes. O código de trânsito autoriza dirigir automóvel com acuidade visual de 20/30 em um olho, ou seja, 39 pacientes em 50 poderiam dirigir sem uso de correção óptica, o que melhora qualidade de vida deles. No presente trabalho, os implantes foram bilaterais, sendo 22 pacientes do grupo de ReSTOR, 8 do grupo Tecnis e 8 do grupo ReZoom. No grupo de ReSTOR, 12 pacientes apresentaram $A V$ 20/20, $\mathrm{J}_{2}$ - J sem correção, $\mathrm{O}$ mesmo ocorreu em 6 pacientes do grupo de ReZoom e 6 do grupo de Tecnis, ou seja,independência ao uso de óculos para todas as atividades, o que reforça que devemos sempre fazer implantes binoculares, assim no grupo ReSTOR, apenas 7 pacientes utilizam óculos de forma esporádica, e no grupo Tecnis e ReZoom 4 pacientes em cada grupo usam óculos para determinadas atividades (uso de computador, jogo de carta, pintar quadro).

Dos 50 pacientes, 27 eram aposentados ou do lar, 3 advogados, 3 engenheiros, 6 empresários, 1 fotógrafo, 1 paisagista, 1 projetista, 1 dentista, 1 tradutor, 1 professor, 1 secretária, 1 modelista, 1 motorista, 1 militar reformado, 1 vigilante.

No estudo multicêntrico japonês ${ }^{(10)}, 136$ pacientes receberam implante de ReSTOR sendo que todos obtiveram acuidade visual sem correção de 20/40 ou melhor, sendo que $88 \%$ deles alcançaram 0.7 ou melhor para longe e 0.4 ou melhor para perto.

Um estudo belga ${ }^{(11)}$, de 104 cirurgias facorefrativas em 52 pacientes présbitas (pacientes com cristalino transparente e présbitas), com implante de LIO Tecnis multifocal, mostrou $95 \%$ dos pacientes lendo $\mathrm{J}_{1} \mathrm{e}$ $100 \%$ lendo J ou melhor sem correção. Observou, também, 20 olhos que necessitaram lasik para correção de erro refrativo pós-implante da LIO multifocal. O trabalho não cita como foi realizado o cálculo biométrico.

Holladay ${ }^{(12)}$ mostrou que em olhos médios as fórmulas são equivalentes.

\section{Conclusão}

A previsibilidade biométrica com uso de novas tecnologias e fórmulas favorece o resultado refracional almejado. No presente estudo,independente da LIO implantada, as três fórmulas apresentaram performance semelhante.

Agradecimentos ao Frederico Rafael Moreira pelo tratamento estatístico do presente estudo.

\section{Abstract}

Purpose: To present biometric predictability, evaluating postoperative refraction and visual acuity in eyes submitted to multifocal intraocular lens implantation. Methods:
Retrospective study in 88 eyes of 50 patients, submitted to phacoemulsification with multifocal lens implant of three commercial brands: ReSTOR, ReZoom and Tecnis. For biometric examination were used: optic biometry associated to the use of tomography of anterior segment and Holladay II biometric formula. With postoperative refraction, visual acuity and implanted IOL, according to Holladay II formula, we calculated the value of IOL to be implanted in case we used SRKT and Haigis formulas. The results were evaluated according to statistical methods. Results: All eyes obtained a visual acuity equal or higher to 20/40 and $J_{3}$ without correction. Holladay II, SRKT and Haigis formulas presented similar performance in all groups. Conclusion: Biometric predictability is improving with the use of optical biometry and last generation formulas.

Keywords: Lenses, intraocular ; Visual acuity; Biometry/methods

\section{REFERÊNCIAS}

1. Centurion V, Lacava AC, Caballero JC. A lente intra-ocular dobrável: cirurgia de catarata por microincisões. In: Padilha MA, organizador. Catarata. Rio de Janeiro: Cultura Médica; 2003.

2. Fine IH, Hoffman RS, Packer M. The new challenge for cataract surgeons. Curr Opin Ophthalmol. 2007; 18(1):1-3.

3. Maximizing patient putcomes with advanced IOL design. Ocular Surgery News [homepage on the Internet]. c2006. [Edition Monograph]. Available from: http://www.osnsupersite.com/ setContent.asp?setid=246 D

4. Bland JM, Altman DG. Statistical methods for assessing agreement between two methods of clinical measurement. Lancet. 1986;1(8476):307-10. Comment in: Lancet. 2004; 363(9403):164; author reply 164-5.

5. Lacava AC, Carrari MJ, Centurion V. A biometria em olhos com comprimento axial médio: estudo comparativo de três fórmulas e a previsibilidade refracional. Rev Bras Oftalmol. 2006; 65(3):157-61.

6. Lacava AC, Centurion V. A biometria em olhos hipermétropes: estudo comparativo de três fórmulas e a previsibilidade refracional. Rev Bras Oftalmol. 2006; 65(3):162-6.

7. Centurion V, Lacava AC, Caballero JC. A performance visual com lente intra-ocular multifocal refrativa AMO Array. Rev Bras Oftalmol. 1999; 58(5):353-9.

8. Leal EB, Lacava AC, Caballero JC, Centurion V. Biometria: interferometria vs ultra-som por aplanação. Rev Bras Oftalmol. 2003; 62(12):872-7.

9. Haigis W, Lege B, Miller N, Schneider B. Comparison of immersion ultrasound biometry and partial coherence interferometry for intraocular lens calculation according to Haigis. Graefes Arch Clin Exp Ophthalmol. 2000; 238(9):765-73.

10. Pedrotti E, Bissen-Miyajima H. High patient satisfaction reported with multifocal IOL. EuroTimes. 2006;11:15.

11. Goes FJ, Scheider A. High-quality functional vision achieved with Tecnis multiocal IOL. EuroTimes. 2006; 11: 12.

12. Holladay JT. Quality of vision: essential optics for the cataract and refractive surgeon. Thorofare: Slack; 2007.
ENDEREÇO PARA CORRESPONDÊNCIA:

$$
\begin{aligned}
& \text { Av. Ibirapuera, } 624 \text { - Ibirapuera } \\
& \text { CEP 04028-000 - São Paulo - SP } \\
& \text { E-mail: centurion@imo.com.br }
\end{aligned}
$$

